\title{
Blood alcohol concentration measurement using a salivary reagent stick: a reliable tool for emergency departments?
}

\author{
I. C. PHAIR, S. MARDEL \& G. G. BODIWALA \\ Accident and Emergency Department, Leicester Royal Infirmary, Leicester.
}

\section{SUMMARY}

Measurements of blood alcohol concentration (BAC) were made on patients who presented to an accident and emergency department with acute alcohol intoxication. A correlation of $r=0.418$ was noted to exist between BAC as measured by sampling saliva and blood.

Blood alcohol concentrations as measured by salivary reagent strip (ALCOSCREEN $^{\mathrm{TM}}$, Chem Elec.) were significantly lower $(p<0.0001)$ than those determined by gas chromatography of serum. Although such reagent strips offer a rapid measurement of BAC, it is concluded that they are unreliable for the quantitative measurement of BAC in accident and emergency departments.

\section{INTRODUCTION}

Attendances to emergency departments by those who are under the influence of alcohol (Yates et al., 1987, Walsh \& Macleod, 1982, Holt et al., 1980) are common. Clinical diagnosis of the level of intoxication in the absence of laboratory measurement is unreliable (Rutherford, 1977).

The use of a breath analyzer offers a speedier measurement of blood alcohol concentration (BAC) than that given by laboratory techniques (Walsh \& Macleod 1982) and a strong correlation with BAC as measured by serum gas chromatography has been reported (Gerberich et al., 1989).

An alcohol reagent strip which estimates BAC by the colour change on a reactive pad when saturated with saliva containing alcohol has been developed (Alco Screen ${ }^{\mathrm{TM}}$,

Correspondence: I. C. Phair FRCS, Senior Registrar, Accident and Emergency Department, Leicester Royal Infirmary, Leicester LE1 $5 W W$, England 
Chem Elec Inc.) A correlation of $r=0.77$ (Williams et al., 1987) and 0.70 (Heller Kaplan 1987) have been reported indicating that BAC measurement using such reagen strips may be sufficiently reliable for clinical practice.

It was therefore thought appropriate to further evaluate the use of such reagent strip in our department.

\section{METHOD}

A series of patients who presented with suspected alcohol intoxication were sample $\Phi$ prospectively. Patients were excluded where there was a suspicion of injury on the basis of history, or clinical examination.

Blood was taken and submitted for analysis by gas chromatography. On admission an estimate of BAC was made by the interpretation of the colour change of the reagent padi. (ALCO-SCREEN) as per the manufacturers instructions.

The examination and assessment of all patients and the interpretation of the reagen? strips were made by the authors. All patients were detained within the department untiP fit for discharge.

Ethical approval was sought and obtained from the Leicester District Health Authority. Statistical analysis where quoted was by the two sample $t$ test.

\section{RESULTS}

A total of 46 patients with a mean age of 33 years (range 16-60 years) were sampled, $3 T$ $(80 \cdot 4 \%)$ were male.

Analysis of results showed that the mean BAC as determined by gas chromatograph of serum was $302.1 \mathrm{mg} \%$, (s.d. 133.6 , range $95-561 \mathrm{mg} \%$ ). The mean BAC as interpreted by salivary reagent strip was $164 \cdot 1 \mathrm{mg} \%$, (s.d. 106.8, range $50-300$ ). The . lower value given by the reagent strips was found to be significant $(p<0.0001)$. correlation of $r=0.418$ existed between BAC as measured by salivary reagent strip and gas chromatography of serum.

The reagent strips give an estimate on a closed scale, the colour change corresponding to BAC of $20,50,100$ or $300 \mathrm{mg} \%$. By grouping the sample into four groups as indicated by reagent strip BAC the scatter of the actual blood alcohol is shown (Fig. 1).

We did not identify any false negative results by using the reagent strips in any of ouro patients.

\section{DISCUSSION}

Jones (1979) found a mean saliva/blood alcohol ratio of 1.077 , with $95 \%$ confidenc 


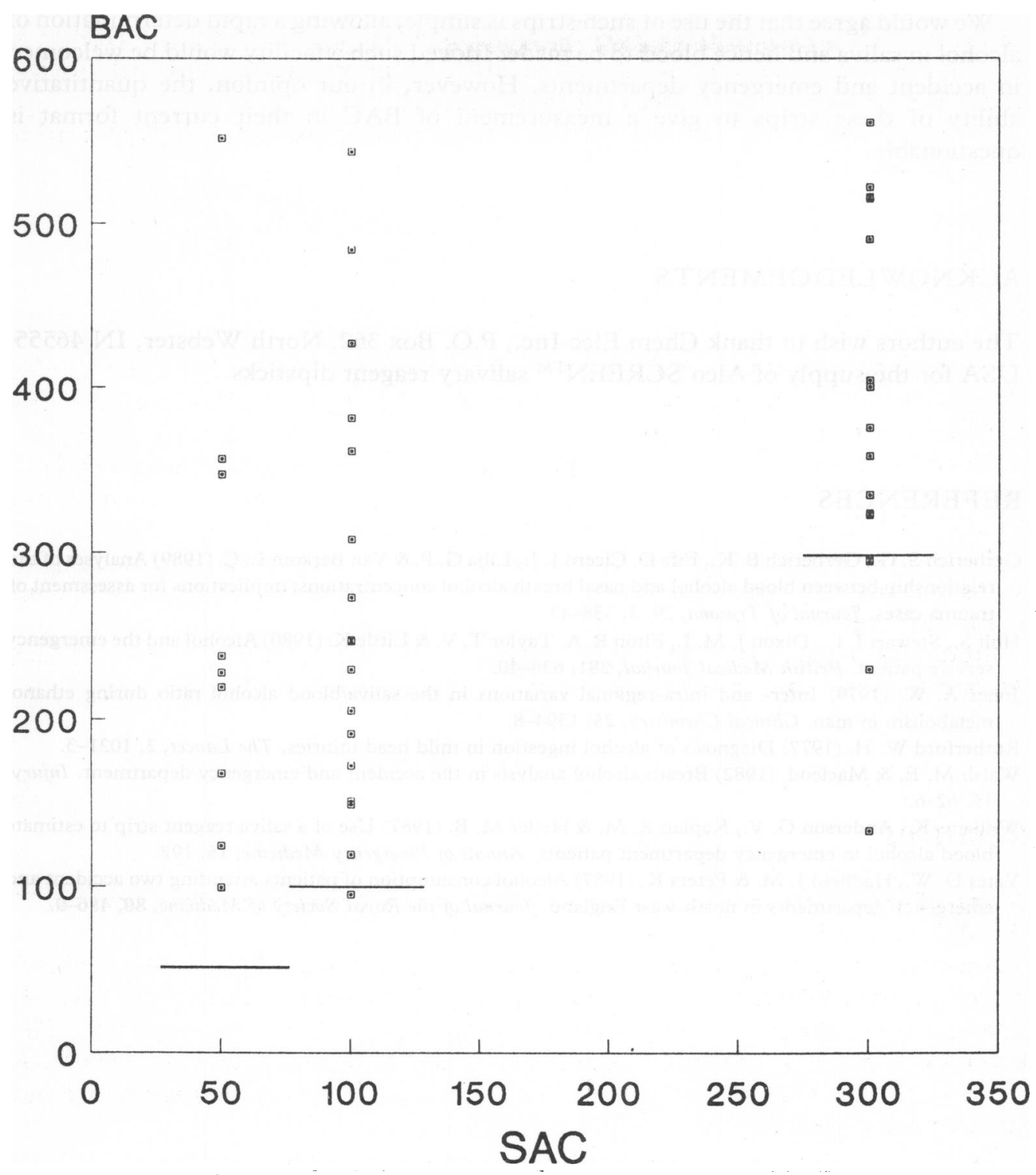

Fig. 1. By grouping the sample into four groups, as indicated by reagent strip BAC the scatter of blood alcohol is shown.

limits of 1.065 and 1.088 and concluded that, the application of saliva as a specimen for blood alcohol determination in clinical and medicolegal work be reconsidered.

The appearance of a salivary alcohol oxidase strip (ALCO SCREEN, Chem Elec) offered the rapid determination of blood alcohol in the emergency situation. Strong correlations of 0.70 (Heller \& Kaplan; 1987) and 0.77 (Williams et al., 1987) suggested that those reagent strips would be useful for emergency departments. 
We would agree that the use of such strips is simple, allowing a rapid determination of alcohol in saliva and hence blood to be made. Indeed such a facility would be welcomed in accident and emergency departments. However, in our opinion, the quantitati ability of these strips to give a measurement of BAC in their current format questionable.

\section{ACKNOWLEDGEMENTS}

The authors wish to thank Chem Elec Inc., P.O. Box 362, North Webster, IN 46555 ,

USA for the supply of Alco SCREEN ${ }^{\mathrm{TM}}$ salivary reagent dipsticks.

\section{REFERENCES}

Gerberich S. G., Gerberich B. K., Fife D. Cicero J. J., Lilja G. P. \& Van Berkom L. C. (1989) Analyses of the relationship between blood alcohol and nasal breath alcohol concentrations; implications for assessment of trauma cases. fournal of Trauma, 29: 3, 338-43. Holt S., Stewart I. C., Dixon J. M. J., Elton R. A. Taylor T. V. \& Little K. (1980) Alcohol and the emergenç $\bar{~}$
service patient. British Medical fournal, 281, 638-40.

Jones A. W. (1979) Inter- and intra-regional variations in the saliva/blood alcohol ratio during ethan metabolism in man. Clinical Chemistry, 25, 1394-8.

Rutherford W. H. (1977) Diagnosis of alcohol ingestion in mild head injuries. The Lancet, 2, 1021-3.

Walsh M. E. \& Macleod. (1982) Breath alcohol analysis in the accident and emergency department. Injur 15, 62-6.

Williams K., Anderson G. V., Kaplan R. M. \& Heller M. B. (1987) Use of a saliva reagent strip to estima blood alcohol in emergency department patients. Annals of Emergency Medicine, 16, 192.

Yates D. W., Hadfield J. M. \& Peters K. (1987) Alcohol consumption of patients attending two accident and emergency departments in north-west England. Fournal of the Royal Society of Medicine, 80, 486-9. 